\title{
Prolonged Expiratory Duration with Elevated Lung Volume in Newborn Infants
}

\author{
ANN R. STARK (17) AND IVAN D. FRANTZ, III \\ Department of Pediatrics, Harvard Medical School, Boston Hospital for Women and Children's Hospital Medical \\ Center, Boston, Massachusetts, USA
}

\begin{abstract}
Summary
End-expiratory lung volume increased $17.5 \pm 5.4 \mathrm{ml}$ (mean \pm SD) in full term infants and $7.7 \pm 2.1 \mathrm{ml}$ in premature infants when 5-7 $\mathrm{cm} \mathrm{H}_{2} \mathrm{O}$ continuous negative pressure (CNEG) was applied around the thorax. In the full term infants, respiratory rate decreased from 52-43 $\mathrm{min}^{-1}(P<0.001$ ), mean inspiratory duration $\left(t_{i}\right)$ was unchanged, and mean duration of expiration $\left(t_{e}\right)$ increased from $0.62 \pm 0.14$ (SE) $-0.84 \pm 0.22 \mathrm{sec}(P<0.001)$ after application of CNEG. $T_{e}$ of occluded efforts on CNEG was also prolonged $(P<0.005)$, although less than $t_{e}$ of spontaneous breaths on CNEG $(P<0.005)$. $\mathrm{T}_{e}$ increased in four of eight premature infants when CNEG was applied. We conclude that phasic vagal feedback regulates $T_{i}$ and $t_{e}$ is controlled by tonic vagal activity.
\end{abstract}

\section{Speculation}

Premature infants may have an optimum functional residual capacity (FRC) which can be achieved with continuous distending pressure, resulting in regularization of respiration.

Clark and von Euler (3), studying the relationship between the depth of a breath and its duration in rebreathing studies on conscious men and anesthetized cats, found that the shortening of inspiration that occurs with increasing tidal volume $\left(V_{T}\right)$ depends on intact vagal feedback. They further showed that the $t_{e}$ depends on the preceding $t_{i}$, although the timing within each breath is independent of the preceding breath. The relationship of tidal volume and respiratory frequency in newborn infants subjected to inspiratory elastic loads and $\mathrm{CO}_{2}$ breathing was examined by Taeusch et al. (11) and Thach et al. (12). The infants showed little decrease in inspiratory time or breath duration when tidal volume increased with $\mathrm{CO}_{2}$ breathing; however, $t_{i}$ increased when tidal volume was diminished by inspiratory loading. In these studies, tidal volume varied, presumably affecting phasic vagal activity, but FRC and, thus, tonic vagal activity, remained relatively constant.

Studies of the effect of elevated resting lung volume (FRC) on respiratory frequency have yielded conflicting results. In anesthetized cats, Grunstein et al. (7) showed that the relationship of $V_{T}$ and $t_{i}$ was independent of end-expiratory lung volume, although $t_{e}$, and, thus, total breath duration ( $\left.t_{\text {tot }}\right)$, was affected by changes in FRC. Similarly, in paralyzed dogs on closed-chest cardiopulmonary bypass, Bartoli et al. (2) showed that an increase in lung volume produced an increase in duration of $t_{e}$, but had little effect on $t_{i}$, as measured from the phrenic electroneurogram. In contrast, D'Angelo and Agostoni (4) reported that increasing lung volume with continuous positive pressure breathing affected both the $\mathrm{V}_{\mathrm{T}^{-}}$ $t_{i}$ relationship and $t_{e}$ in anesthetized rabbits, cats, and dogs. In human, premature infants with hyaline membrane disease, increasing lung volume with continuous distending pressure produced a decrease in respiratory frequency (1), although this change was not seen in healthy premature infants (10). In neither study was the effect on $t_{i}$ and $t_{e}$ specifically examined. The purpose of the present study was to quantify the effects of static lung volume changes on respiratory rate and inspiratory and expiratory duration, both with and without the influence of phasic vagal activity, in a group of normal, full term and premature infants.

\section{MATERIALS AND METHODS}

Fifteen normal, full term infants (birth weight, $3.40 \pm 0.35 \mathrm{~kg}$, mean $\pm S D$ ) and eight healthy, premature infants of 32-34 wk gestation (birth weight, $1.67 \pm 0.21 \mathrm{~kg}$ ) were studied during the first 2-4 days of life, with the exception of one premature infant studied on day 9 . Informed consent for the study was obtained from the parents and pediatrician of each infant. Measurements were made while the infants were sleeping quietly in the supine position, enclosed below the neck in a negative pressure box. This box was a plastic enclosure with an inflatable latex neck seal and side portholes for access to the infant. Negative pressure was applied to the box by a vacuum cleaner motor attached by a threeway valve. In the premature infants, skin temperature was maintained at $36.5^{\circ}$ with a servocontrolled radiant warmer.

All measurements were obtained for each infant before and after application of 5-7 $\mathrm{cm} \mathrm{H}_{2} \mathrm{O}$ CNEG to the box. Air flow was measured with a heated Fleisch pneumotachograph (no. 00) and Statham PM 15E pressure transducer while the infant breathed through a face mask, and flow was integrated electronically to give volume. Mask pressure was measured with a Hewlett-Packard 270 transducer. The dead space of the system was approximately $6 \mathrm{ml}$. All signals were recorded on a Beckman polygraph.

The measurements were made during quiet, regular breathing. The increment in lung volume that occurred with application of CNEG was measured from the integrated flow signal as the difference between the volume at end-expiration during control breathing and that at the increased volume. Usually the new resting lung volume was achieved within $2-3$ breaths after CNEG was applied. Ten single breath occlusions of the airway were done at end-expiration in each infant before and after application of CNEG, allowing a 30-60 sec recovery period between occlusions. Tidal volume and breath duration were measured from breaths preceding each occlusion. $T_{i}$ and $t_{\text {tot }}$ were measured between points of zero flow. $T_{e}$ was then obtained by subtraction. Percent $t_{e}$ was calculated as $t_{e} / t_{t o t} \times 100 \%$. For the occluded breaths, $t_{i}$ was measured on the pressure tracing from the start of the occlusion to the point of peak inspiratory effort, and $t_{e}$ was measured from the end of $t_{i}$ to the start of the next inspiratory effort. Results were tested for statistical significance using the paired $t$ test. 


\section{RESULTS}

\section{FULL TERM INFANTS}

When 5-7 $\mathrm{cm} \mathrm{H}_{2} \mathrm{O}$ negative pressure was applied around the thorax, end-expiratory lung volume increased $17.5 \pm 5.4 \mathrm{ml}$ (mean $\pm S D$ ), a value consistent with reported compliance measurements. Tidal volume did not change significantly. Respiratory rate decreased from 52-43 $\mathrm{min}^{-1}(P<0.001)$. Schematic representations of average breaths on and off CNEG are shown in Figure 1 and individual values are contained in Table 1 . Mean $t_{i}$ was unchanged after application of CNEG. Mean $t_{e}$ increased from $0.62 \pm 0.14$ $(\mathrm{SE})-0.84 \pm 0.22 \mathrm{sec}(P<0.001)$. Percent $\mathrm{t}_{\mathrm{e}}\left(\mathrm{t}_{\mathrm{e}} / \mathrm{t}_{\mathrm{t} \text { to }} \times 100\right)$ also increased in 14 of the 15 infants. The effect of CNEG on breath duration was immediate, and there was no change in the effect during the approximately $20 \mathrm{~min}$ period of CNEG. Timing immediately returned to control values when CNEG was discontinued (Fig. 2). End-expiratory occlusion of the airway resulted in significant prolongation of the inspiratory effort, as has been described previously $(9,11)$. The occluded $t_{i}$ was similar before and after application of CNEG. Occlusion did not affect expiratory duration. $T_{e}$ of occluded efforts on CNEG was shorter than $t_{\mathrm{e}}$ of spontaneous breaths on CNEG $(P<0.005)$, but still longer than $t_{\mathrm{e}}$ of spontaneous breaths off CNEG $(P<0.005)$.

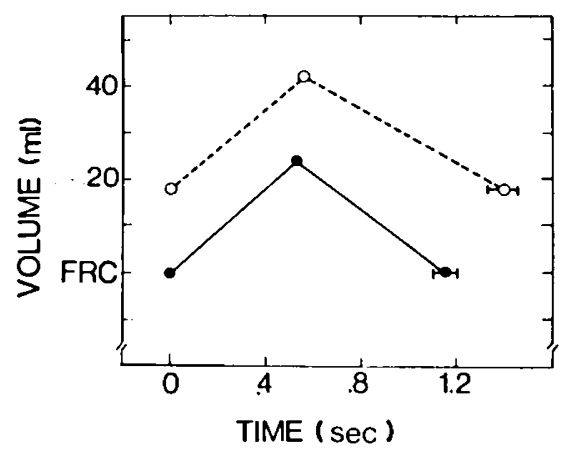

Fig. 1. Representation of breaths off (solid line) and on (broken line) CNEG. The confidence limits indicated are \pm 1 SE. Note that while CNEG increases FRC an amount almost equivalent to tidal volume, $t_{i}$ is not shortened. There is however, a prolongation of $t_{e}$ on CNEG.

\section{PREMATURE INFANTS}

Resting lung volume increased $7.7 \pm 2.1 \mathrm{ml}$ when CNEG was applied to the premature infants, also consistent with reported compliance values. As in the full term infants, tidal volume was not affected. The effects on timing were less consistent in the premature than in the full term group (Table 2). Mean respiratory rate, $58 \mathrm{~min}^{-1}$, was not changed. Inspiratory duration was affected little in six of the eight infants. In four of the infants, $t_{e}$ increased when CNEG was applied, while it was essentially unchanged in the other four infants. The effect on $t_{e}$ was not associated with the magnitude of lung volume increase, nor with the initial respiratory rate. Airway occlusion in the premature infants resulted in prolongation of $t_{i}$, both on and off CNEG. As in full term infants, $t_{e}$ was not affected by occlusion.

\section{DISCUSSION}

We have shown in newborn infants that an increase in resting lung volume of nearly $20 \%$ of estimated FRC, or approximately equivalent to tidal volume, results in significant prolongation of $t_{e}$, with little effect on $t_{i}$ or $V_{T}$. Elimination of phasic lung volume change by airway occlusion results in lengthening of $t_{i}$, as previously shown $(9,11)$, but this effect is not influenced by static lung volume changes. Our findings support the hypothesis that $t_{\mathbf{e}}$ is influenced by changes in FRC, while $t_{i}$ is affected by phasic lung volume changes. We conclude from our data that phasic lung

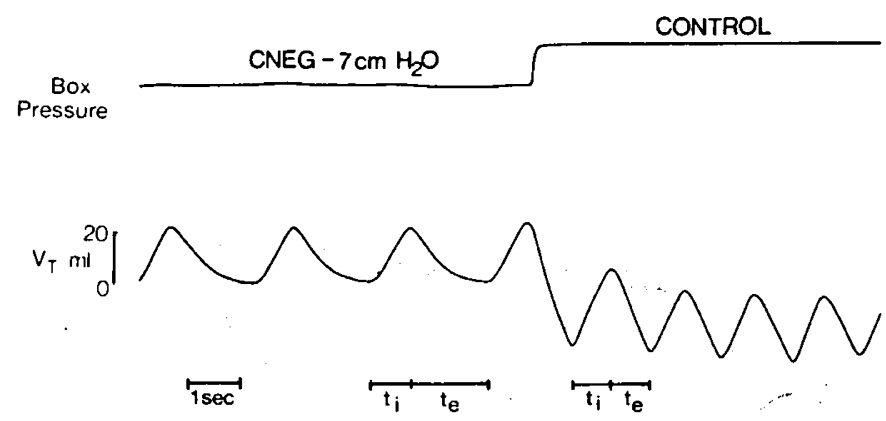

Fig. 2. Sample tracing from an infant as box pressure is changed from $-7 \mathrm{~cm} \mathrm{H}_{2} \mathrm{O}$ to atmospheric. Note the change in end-expiratory lung volume and shortening of $t_{e}$ which occur simultaneously with the change in box pressure.

Table 1. Effect of CNEG and occlusion on respiratory timing in full term infants

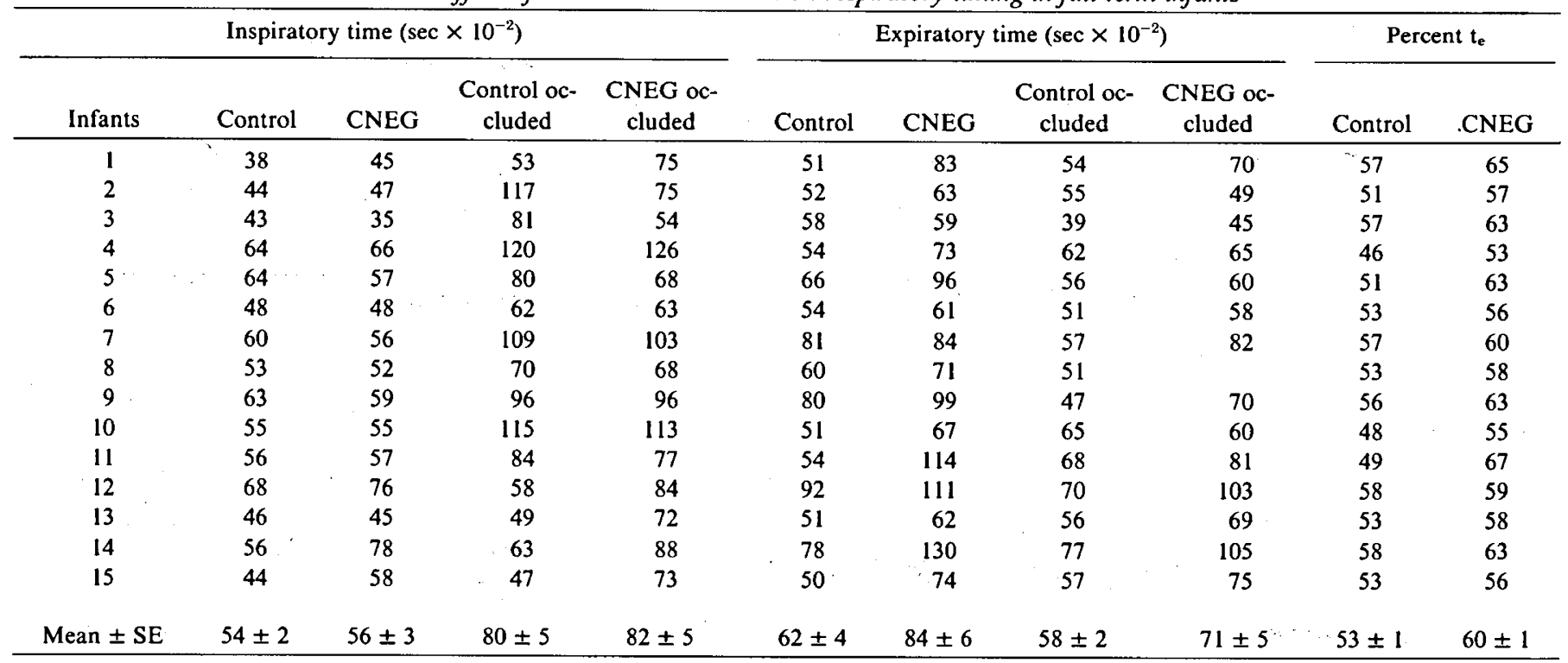


Table 2. Effect of CNEG and occlusion on respiratory timing in premature infants

\begin{tabular}{|c|c|c|c|c|c|c|c|c|c|c|}
\hline \multicolumn{5}{|c|}{ Inspiratory time $\left(\sec \times 10^{-2}\right)$} & \multicolumn{4}{|c|}{ Expiratory time $\left(\sec \times 10^{-2}\right)$} & \multicolumn{2}{|c|}{ Percent $t_{e}$} \\
\hline 17 & 33 & 29 & 82 & 60 & 35 & 38 & 46 & 39 & 51 & 57 \\
\hline 18 & 46 & 40 & 55 & 68 & 55 & 52 & 74 & 58 & 54 & 57 \\
\hline 19 & 54 & 50 & 61 & 69 & 83 & 81 & 81 & 104 & 61 & 62 \\
\hline 22 & 43 & 47 & 64 & 70 & 51 & 58 & 69 & 55 & 54 & 55 \\
\hline 23 & 38 & 30 & 33 & 24 & 59 & 65 & 41 & 30 & 61 & 68 \\
\hline
\end{tabular}

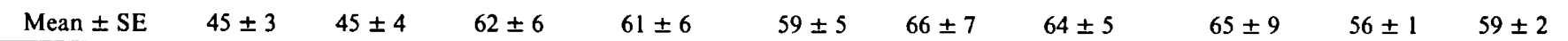

feedback regulates $t_{i}$ and that $t_{e}$ is controlled by tonic vagal activity.

This condition is illustrated schematically in Figure 3. In Figure 3 (left panel) the open circle represents $t_{i}$ in the absence of phasic vagal activity (i.e., after airway occlusion at FRC). When the infant takes a normal breath, $t_{i}$ is shortened (closed circle). When a new elevated FRC is achieved with CNEG, $t_{i}$ in the absence of phasic vagal activity (open triangle) is unchanged from that at the control lung volume. When a breath is taken (closed triangle) $t_{i}$ is shortened to the same extent as before CNEG was applied. The difference in $t_{i}$ before and after occlusion (open $v$ s. closed symbols) represents the effect of phasic vagal activity on $t_{i}$. On the other hand, changing FRC by an amount approximately equal to tidal volume has no effect on $t_{i}$, indicating an absence of influence on tonic vagal effect on $t_{i}$.

Figure 3 (right panel) is a similar representation for $t_{e}$. In this case, phasic vagal activity has little effect; that is, there is little change in $t_{e}$ from the occluded to the unoccluded state. However, increasing tonic activity by increasing lung volume (moving from circles to triangles) results in a prolongation of $t_{e}$. The occluded $t_{e}$ on CNEG, although prolonged, is significantly shorter than unoccluded $t_{e}$ on CNEG. This may be secondary to blood gas changes occurring during occlusion. Evidence to support this comes from the studies of Frantz and Milic-Emili (5) and Younes et al. (14) who have observed that when airway occlusion at FRC is maintained for multiple inspiratory efforts, a progressive increase in pressure is generated. This pressure increase can be attributed to blood gas changes and is apparent by the second occluded effort, which occurs 1.5-2 sec after occlusion. Blood gas changes may likewise affect $t_{e}$ to cause premature shortening.

The von Euler (3) concept of the respiratory control system states that $t_{i}$ is decreased at increased lung volume, and that $t_{e}$ is dependent on $t_{i}$. Our results appear to be in conflict with this concept. That $t_{i}$ was not shortened by the application of CNEG can be explained in three ways. First, the increase in volume may have been insufficient to get the infant out of von Euler's range 1 $\left(t_{i}\right.$ independent of $V_{T}$ ) into a range where significant shortening of $t_{i}$ would occur. Second, vagal stretch receptors may already be maximally stimulated at resting tidal volume so that a further increase in lung volume would evoke no additional response. Third, there may be a resetting of tonic vagal activity when FRC is increased, thus moving the entire $V_{T}-t_{i}$ relationship upward. Rebreathing and elastic loading data $(6,9,10)$ imply that either of the first two possibilities could be correct. That is, increasing tidal volume by $2-3$ fold by $\mathrm{CO}_{2}$ rebreathing did not, on the average, result in a decrease in $t_{j}$. Decreasing tidal volume with elastic loads did, however, cause prolongation of $t_{i}$, implying that infants are maximally stimulated at resting tidal volumes. Since $t_{i}$ after occlusion in our study was the same before and after CNEG was applied, we conclude that the third possibility is correct. That is, increasing FRC as opposed to tidal volume, results in a resetting of tonic stretch receptors and shifts the $\mathrm{V}_{\mathrm{T}}-\mathrm{t}_{\mathrm{i}}$ relationship upward.

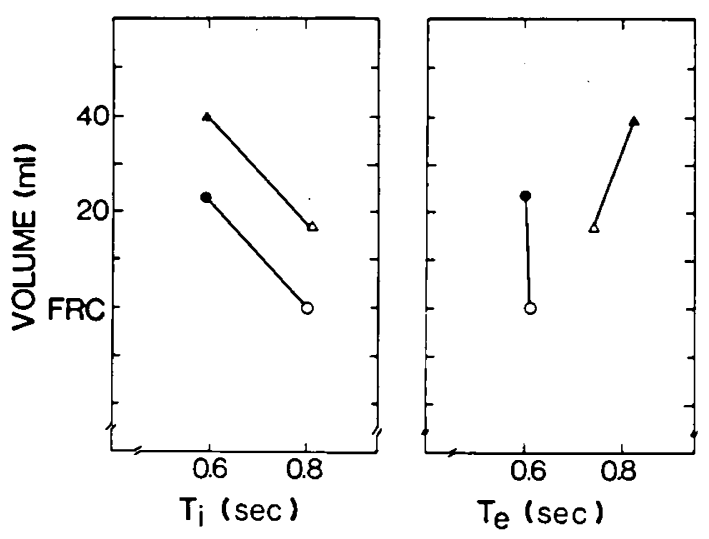

Fig. 3. Comparison of $t_{i}$ and $t_{e}$ on (triangles) and off (circles) CNEG with (open symbols) and without (closed symbols) airway occlusion. Note from the left panel that occlusion results in a marked prolongation of $t_{i}$ but that CNEG has little effect. In the right panel, note the minimal effect on $t_{e}$ of occlusion, and the pronounced prolongation resulting from CNEG.

Furthermore, inasmuch as $t_{e}$ was prolonged on CNEG while $t_{i}$ was unaffected, it is apparent that $t_{e}$ is not necessarily dependent on $t_{i}$. Tonic stretch receptor stimulation must act to prolong $t_{e}$ independent of effects on $t_{i}$.

The variability seen in the results obtained in premature infants may be part of the inherent variability of respiration in these infants. Sleep state, not controlled in this study, may also be a factor. Another possibility is that there is an optimum FRC for appropriate respiratory timing in premature infants, and that these infants have lung volumes that vary in relationship to the optimum. Those infants who demonstrated a prolongation of $t_{e}$ and a decrease in minute ventilation may have been brought to optimum FRC when CNEG was applied. Other infants who did not respond may have been at or near optimum FRC before CNEG was applied or may have been too far below it for the amount of CNEG.administered to have been effective. Bartoli et al. (2) have shown in dogs that vagotomy can have variable effects on the lung volume-expiratory time relationship, depending on the FRC before vagotomy was done. They postulate that there is a neutral point for FRC and different results can be obtained if vagotomy is done at lung volumes on either side of the neutral point. This neutral point may be similar to the optimum FRC we have postulated for the preterm infants in our study.

Increasing FRC in preterm infants has been shown to decrease irregularity of respiration. FRC is lower in premature than term infants and has been shown to decrease over the lst wk of life (13), a time when respiratory irregularity (apnea and periodic breathing) is at a maximum. Infants whose FRC is brought to optimum may have the most prominent decrease in apneic spells 
when continuous distending pressure is used to regularize respiration.

We conclude from our studies that phasic vagal influence on inspiration in the neonate is at a maximum in the normal tidal volume range, and that increasing resting lung volume results in a resetting of tonic stretch receptors so that $t_{i}$ does not change. $T_{e}$ can be influenced by changes in tonic vagal activity in the absence of effects on $t_{i}$, indicating that $t_{i}$ and $t_{e}$ are not necessarily coupled. Infants may have an optimum FRC which can be achieved with continuous distending pressure, resulting in a decrease in minute ventilation and regularization of respiration.

\section{REFERENCES AND NOTES}

I. Bancalari, E., Garcia, O., and Jesse, M. J.: Effects on continuous negative pressure on lung mechanics in idiopathic respiratory distress syndrome. Pediatrics, 51: 485 (1973).

2. Bartoli, A., Bystrzycka. E., Guz, A., Jain, S. K., Noble, M. I. M., and Trenchard, D.: Studies of the pulmonary vagal control of central respiratory rhythm in the absence of breathing movements. J. Physiol., 230: 499 (1973).

3. Clark, F. J., and von Euler, C.: On the regulation of the depth and rate of breathing. J. Physiol., 222: 267 (1972).

4. D'Angelo, E., and Agostoni, E.: Tonic vagal influences on inspiratory duration. Resp. Physiol.. 24: 287 (1975).

5. Frantz, I. D., and Milic-Emili, J.: The progressive response of the newborn infant to added respiratory loads. Resp. Physiol., 24: 233 (1975).

6. Frantz, I. D., Adler, S. M., Thach, B. T., and Taeusch, H. W.: Maturational effects on respiratory responses to carbon dioxide in premature infants. J. Appl. Physiol., 41: 41 (1976).

7. Grunstein, M. M., Younes, M., and Milic-Emili. J.: Control of tidal volume and respiratory frequency in anesthetized cats. J. Appl. Physiol., 35: 463 (1973).

8. Martin, R. J., Okken, A., Katona, P., and Klaus, M.: Evidence for a tonic component of the Hering-Breuer inflation reflex in the term neonate. (abstract) Pediatr. Res., 11: 575 (1977).

9. Olinsky, A., Bryan, M. H., and Bryan, A. C.: Influence of lung inflation on respiratory control in neonates. J. Appl. Physiol., 36: 426 (1974).

10. Saunders, R. A., Milner, A. D., and Hopkin, I. E.: The effects of continuous positive airway pressure on lung mechanics and lung volumes in the neonate. Biol. Neonate. 29: 178 (1976).

11. Taeusch, H. W., Carson. S., Frantz, I. D., and Milic-Emili, J.: Respiratory regulation after elastic loading and $\mathrm{CO}_{2}$ rebreathing in normal term infants. J. Pediatr., 88: 102 (1975).

12. Thach, B. T., Frantz, I. D., Adler, S. M., Taeusch, H. W., and Avery, M. E.: Vagal influence on inspiratory duration in premature infants: absence of postnatal or gestational age-related differences (abstract). Fed. Proc., 34: 365 (1975).

12. Thibeault. D. W., Wong. M. M., and Auld, P. A. M.: Thoracic gas volume changes in premature infants. Pediatrics, 40: 403 (1967).

14. Younes, M., Arkinstall, W., and Milic-Emili, J.: Mechanism of rapid ventilatory compensation to added elastic loads in cats. J. Appl. Physiol., 35: 443 (1973).

15. Dr. Stark is an E. L. Trudeau Fellow of the American Lung Association.

16. Dr. Stark and Dr. Frantz are recipients of Young Investigator Awards from the National Heart. Lung, and Blood Institute.

17. Requests for reprints should be addressed to: Dr. Ann R. Stark, Joint Program in Neonatology, 221 Longwood Avenue, Boston, MA 02115 (USA).

18. Received for publication February 6, 1978.

19. Accepted for publication May 15, 1978. 